Review

\title{
Cancer Stem Cells: Biological Functions and Therapeutically Targeting
}

\author{
Marius Eugen Ciurea ${ }^{1, \dagger}$, Ada Maria Georgescu ${ }^{1, \dagger}$, Stefana Oana Purcaru ${ }^{1}$, \\ Stefan-Alexandru Artene ${ }^{1}$, Ghazaleh Hooshyar Emami ${ }^{1}$, Mihai Virgil Boldeanu ${ }^{2}$, \\ Daniela Elise Tache ${ }^{1}$ and Anica Dricu ${ }^{1, *}$
}

1 Faculty of Medicine, University of Medicine and Pharmacy of Craiova, Str. Petru Rares nr. 2-4, Craiova 710204, Romania; E-Mails: meciurea@gmail.com (M.E.C.); ada_georgescu@yahoo.com (A.M.G.); stoapo@yahoo.com (S.O.P.); stefan.artene@yahoo.com (S.-A.A.); ghazaleh_ei@yahoo.co.uk (G.H.E.); dodolita@yahoo.it (D.E.T.)

2 Stem Cell Bank Unit, Medico Science SRL, Str. Brazda lui Novac nr. 1B, Craiova 200690, Romania; E-Mail: info@medicoscience.ro

$\dagger$ These authors contributed equally to this work.

* Author to whom correspondence should be addressed; E-Mail: anica.dricu@live.co.uk or anica.dricu@webmailumfcv.ro; Tel.: +40-0351-443-500; Fax: +40-0251-593-077.

Received: 23 March 2014; in revised form: 20 April 2014 / Accepted: 24 April 2014 / Published: 9 May 2014

\begin{abstract}
Almost all tumors are composed of a heterogeneous cell population, making them difficult to treat. A small cancer stem cell population with a low proliferation rate and a high tumorigenic potential is thought to be responsible for cancer development, metastasis and resistance to therapy. Stem cells were reported to be involved in both normal development and carcinogenesis, some molecular mechanisms being common in both processes. No less controversial, stem cells are considered to be important in treatment of malignant diseases both as targets and drug carriers. The efforts to understand the role of different signalling in cancer stem cells requires in depth knowledge about the mechanisms that control their self-renewal, differentiation and malignant potential. The aim of this paper is to discuss insights into cancer stem cells historical background and to provide a brief review of the new therapeutic strategies for targeting cancer stem cells.
\end{abstract}

Keywords: cancer stem cell; tumorigenicity; signalling pathways; cancer stem cell markers; cancer therapy 


\section{Introduction}

According to the modern theory of carcinogenesis, malignant transformation may occur due to the action of a wide range of mutagenic agents on stem cells present in the adult tissue [1]. The exact origin of cancer stem cells (CSCs) remains unknown, despite intensive research in the last decade. In a study on leukemia, published by Bonnet et al. [2] in Nature Medicine in 1997, the existence of a heterogeneous tumor cell population was first mentioned; this cell population was analyzed in terms of proliferation and differentiation. These cells, found in leukemia cell populations, were thought to have stem cells properties, such as self-renewal capacity and high proliferation rate [3]. Another study conducted by Passegué et al. [4] demonstrated that in leukemia, the presence of stem cells is necessary and sufficient for maintaining the tumor cell population. It has also been suggested that the unlimited self-renewal capacity of CSCs may be the cause of tumor recurrence [5]. It has recently been demonstrated that CSCs are present in both hematologic malignancies and solid tumors (i.e., breast cancer, brain tumors, malignant melanoma or prostate cancer) [6,7]. Additionally, surface markers of the CSCs have been identified in many types of cancers including: leukemia CD34+/CD38-, breast cancer CD44+/ESA+/CD24-, brain cancer CD133+, multiple myeloma CD138-, pancreatic cancer CD44+/CD24+/ESA+, colon cancer CD133+, liver cancer CD133+ [5], prostate cancer CD44+/CD133+ [6], lung cancer CD133+ and ovarian cancer CD133+/CD44+/CD117+ [7]. After numerous preclinical and clinical studies, it has been shown that adult stem cells could turn into CSCs with specific surface markers $[8,9]$. Specific targeting of tumor stem cells has been suggested to be a good alternative for cancer treatment $[10,11]$. Some studies demonstrate that CSCs exist in primary human sarcoma tumors such as bone sarcomas [12], and CD133 has been shown to be a potential marker for identification of the CSCs as seen in a paper by Suva et al. [13] where a population of Ewing's sarcoma family tumor (ESFT) cells expressed CD133 which also fulfilled in vivo criteria of CSCs and in vitro plasticity properties of mesenchymal stem cells $[12,13]$.

\section{Tumor Cells vs. Tumor Stem Cells}

Over the years, a variety of polemical concepts have been generated to explain the process of carcinogenesis. In the early 1900s, scientists first believed that cancer is a somatic cell disorder [14] and soon after Tyzzer, E. introduced the notion of "somatic mutation" in connection with cancer [15]. However, Boveri's observation [14] was crucial in understanding the process of carcinogens. He believed that chromosomal abnormalities are fundamental to cancer development, anticipating the cancer genetic hypothesis [14]. More convincing arguments and evidence to sustain the cancer genetic hypothesis came from the discovery that chemicals and radiations could act as mutagenic factors $[16,17]$.

The cancer genetic hypothesis was further supported by Knudson's two-hit theory, postulating that at least two genetic mutations in a tumor suppressor gene are necessary to generate cancer [18]. Two-hit hypothesis of carcinogenesis may explain why people with a family history of cancer do not necessarily develop malignancies. These individuals may inherit a mutated gene, but at least a second mutation is needed for occurrence of cancer. This theory may also explain why people with no family history of cancer can develop cancer, as long as there are at least two genetic mutations that may occur for a variety of reasons $[19,20]$. In support of the two mutation theory, other clinical observations showed 
that somatic mutations in the retinoblastoma gene were present in patients with several types of cancer (e.g., sarcomas breast cancer, bladder cancer, lung cancer) [21,22].

In 1976, Nowell, P.C. proposed the multistep genetic model of tumorigenesis [23] and in 2000, Hanahan and Weinberg explained the classical model of molecular transformation in cancer cells [24]. These studies defined the model of carcinogenesis known as the "somatic mutation theory", stating that cancer is a clonal, cell-based disease, assuming that quiescence is the regular state of cells in the body [24,25].

The "somatic mutation theory" has dominated oncology for more than 40 years; it explains that multistep genetic alteration of recessively acting tumor suppressor genes and dominantly acting oncogenes take place in cells of origin, giving rise to tumor proliferation, invasion, metastasis and drug resistance.

However, the cellular origin of cancer and the mechanisms behind cancer development are still debatable since tumors, be they solid or liquid, are heterogeneous cell populations composed of a large number of tumor and non-tumor cell populations. From this perspective, a new model - the tissue organization field model - tries to explain the development of cancer, meaning that cancer is a tissue-based disease and involves a dynamic communication between the various cell populations coexisting in cancer tissue and also stroma/epithelium interactions [26,27]. These models tried to define the model of carcinogenesis, responsible for both clonal selection and tumor cell heterogeneity.

Recently, in a study by Feinberg et al. the epigenetic aspect was added to this theory which accounts for the alterations in global DNA methylation that in turn can induce both abnormal activation of proliferation genes and tumor suppressor genes silencing [28]. In addition, the author suggested that "tumor-progenitor genes" promote epigenetic disruption of stem/progenitor cells and that the epigenetic plasticity together with genetic injuries are responsible for tumor cell heterogeneity and tumor progression [28].

Most of current understanding about the existence of stem/progenitor cells in adult tissue originates from animal model studies [29-31], many authors suggesting that the existence of stem/progenitor cells and the committed progenitors or transit-amplifying cells may enable the malignant transformation [32].

The CSCs theory is an old idea that was first described in 1973 by Moore et al. [33]. According to the current CSCs accepted hypothesis, besides the cells that form the tumor bulk, the malignant transformation also involves the existence of a cell population with special properties such as self-healing ability, called cancer stem cells or tumor stem cells [34-36].

CSCs are able to self-heal and sustain tumor growth and heterogeneity. In this context, the CSCs theory has many similarities to the evolution model of whole body. Stem cells that pass through embryogenesis can be normal adult stem cells (tissue stem cell) or differentiated cells. Due to similarities with the evolution model of whole body, it is speculated that an adult stem cell that acquires a genetic mutation, develops into a CSC of origin (CSCO) that acquires several new genetic mutations, developing into a CSC and finally giving rise to cancer [37].

The CSC hypothesis gained credibility because all main cancer-origin theories (genetic/epigenetic events, chemical-, infection-, virus-induced carcinogenesis) indicated that the tissue stem cell is involved in the generation of cancer [2,38-43]. 
Unlike the traditional theory of carcinogenesis, recent results obtained from different research groups suggest that CSCs are the driving force of tumorigenesis and metastases, and are the cause of tumor properties, such as proliferation, aggressiveness and resistance to treatment [44].

To explain how cancer occurs and progresses from CSCs, the hierarchy model, also known as clonogenic model, has been proposed. According to the CSC clonal model, only a subset of cancer cells called CSCs are able to initiate malignant progression resulting in heterogeneous tumors (Figure 1) [45-47].

Figure 1. Theory of malignant transformations from adult stem cells. On the left, the stochastic model postulates that several cancer cell types have an equal ability to regenerate and proliferate, each one being capable of giving birth to another tumor (the green, pink, blue and red cells represent similar, non-stem tumoral cell populations); On the right, the hierarchy model postulates that only a specific population (cancer stem cells represented by the red cells marked with a CSC in the middle) has the distinct ability to regenerate, multiply and to differentiate into other subset populations (the green, blue and pink cells), hence giving birth to other tumors.

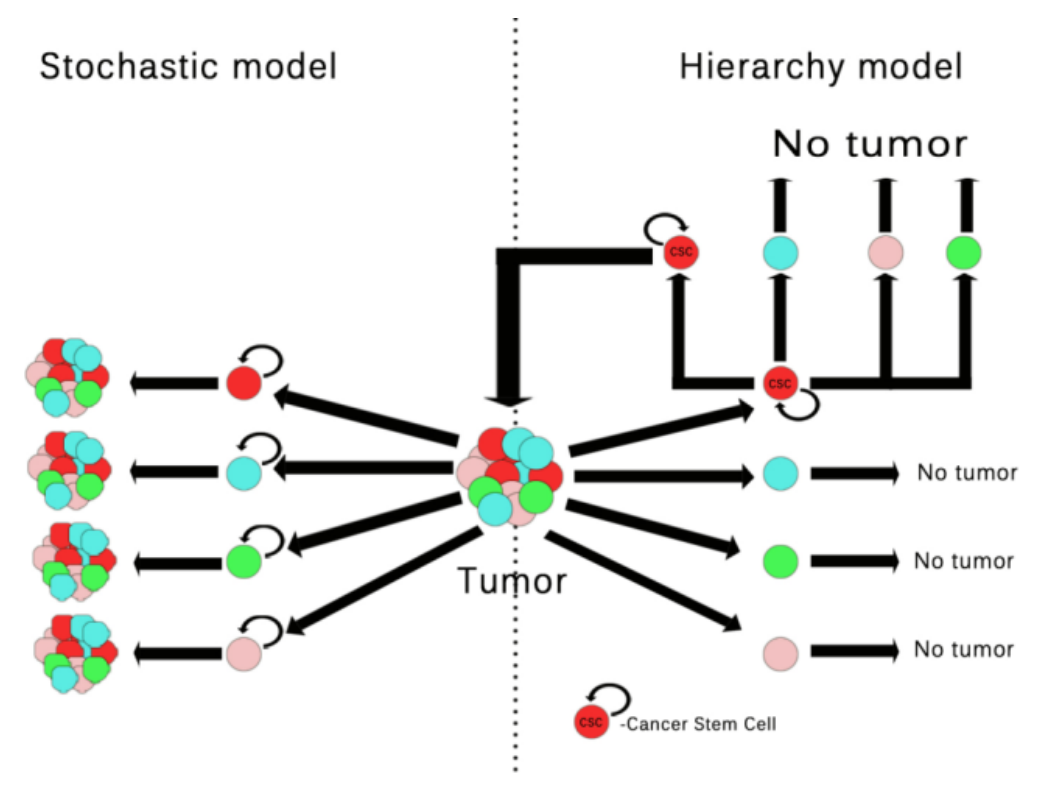

In a study by Chaffer et al., the authors demonstrated that both normal and CSC-like cells can materialize de novo from more differentiated cell types. This discovery brings an addition to the hierarchical models that do not take into consideration a special type of cell plasticity in which stem and non-stem states are converted into one another, often as the result of the cell environment [48]. Consequently, researchers have used the stochastic model to illustrate the CSC model of cancer development, whereby particular circumstances in a mix-tumor cell population transform any tumor cell into a stochastically tumor-initiating cell, leading to tumor heterogeneity (Figure 1) [44,49-51].

Tissue organization architecture model and external stimuli were suggested to be important in CSC initiating tumors. In the paper of Vermeulen et al., the authors demonstrated that in colon CSCs, Wnt activity and cancer stemness may be regulated by environmental stimuli. They also showed that by reprogramming, clonogenic differentiated cancer cells can no longer turn into CSCs, and retrieve their tumorigenic capacity when stimulated with myofibroblast-derived factors, suggesting that cancer stemness is not an unyielding characteristic [52]. 
Roesch et al. detected a slowly dividing cell population and demonstrated that it could sustain melanoma growth and self-renew. They also showed that these cells can shift cell state through epigenetic changes mediated by JARID1B, implying cell plasticity [53].

The cancer associated fibroblasts (CAFs) and the epithelial-mesenchymal transition (EMT) have also been reported as important components of the tumor microenvironment induced cell plasticity. The current reports of the CAFs acknowledge that this stimulates cancer cells to express stem cell markers like CD133 or CD44, and stimulate local acidification that in turn alters the extracellular matrix pattern, increasing cancer cell anchorage-independent growth potential and their tumor-repopulating ability [54-56].

EMT is a key factor that is often activated during embryogenesis but also during cancer invasion and metastasis [57,58]. Mani et al. [59] showed that "Induction of an EMT in non-tumorigenic and immortalized human mammary epithelial cells results in the acquisition of mesenchymal traits and properties associated with mammary epithelial stem cells". These findings illustrate a direct link between the EMT and the acquisition of epithelial stem-cell properties [59]. Interestingly, the mechanisms that promote the CAFs and EMT reactivity in cancer cells have been shown to be analogous, both enrolling redox related molecules such as hypoxia inducible factor 1 (HIF) and cyclooxygenase 2 (COX2) [60]. In addition, CAFs were shown to induce EMT by an epigenetic mechanism, which in turn disrupts cancer cells adhesive cell-to-cell interactions, acquiring a mesenchymal motility and escaping from primary neoplastic lesions and metastasis [54,61].

The proposal that CSC are involved in carcinogenesis is consistent with the identification of CSC subpopulation in leukemia [3,4,62], breast cancer [63], prostate cancer [8], ovarian cancer [7] and more recently, in certain types of brain tumors [64].

The percentage of CSCs identified in the whole tumor cell population was not the same in different tumor types $[50,65]$ and the number of the CSCs in the tumor was reported to be correlated with patient prognosis $[66,67]$. However, other groups showed that tumors of the same histological type contain a very different percentage of CSCs, varying from $0.03 \%$ to approximately $100 \%$ determined by different techniques involving antibodies against surface markers such as CD133+ or using Hoechst dye [68-71], suggesting that the number of the CSCs is of doubtful prognostic value. Other studies using sphere cultures and differentiation identified CD133+ CSCs in osterosarcoma-stabilized cell line by showing that only $\mathrm{CD} 133+$ cells were able to form sarcospheres. Whereas, Aldehyde Dehydrogenase (ALDH) assays are also used to identify populations of CSCs within breast, colon and lung cancer but are also rarely used for identifying osteosarcoma cells [12].

Because CSCs divide slowly, it has also been suggested that the CSC population is responsible for tumor resistance to treatment.

\section{Molecular Signalling Pathways in Cancer Stem Cells}

Little is known about the relationship between CSCs and other heterogeneous cell populations within a certain tumor and even less is known about signalling pathways that these cells use in order to coordinate their behaviour. Considered "The Holy Trinity" of cell molecular signalling, Notch, Hedgehog and Wnt pathways along with the B lymphoma Mo-MLV (Moloney murine leukaemia virus) insertion 
region 1 homolog polycomb ring finger oncogene known as BMI-1 pathway, are currently the most studied molecules [72].

The Notch pathway plays a major role in both normal and CSCs. Once activated by its ligands (Delta-like and Jagged ligands), Notch receptor translocates to the nucleus and associates with a DNA-bound protein, starting a cascade of transduction events in the cell [73]. In the normal stem cell population, Notch receptor is important in cellular fundamental functions such as proliferation, differentiation and apoptosis. The deregulation of Notch pathway results in abnormal proliferation, reduced differentiation and arrested apoptosis with major implications in a variety of cancers such as: T-cell acute lymphoblastic leukemia, melanoma, breast cancer, meningioma and lung adenocarcinoma $[74,75]$.

Hedgehog is a molecule responsible for a plethora of effects on the early development of different parts of the body. The Hedgehog pathway was first discovered by experimental biologists in the Drosophila Melanogaster fly [76]. After further research, three different homologues were discovered in the human body, each of them having a distinct role: Sonic Hedghehog (SHH), the Desert Hedgehog (DHH) and the Indian Hedgehog (IHH) [76]. Present in both embryo and adult stem cells, the SHH is involved in the development of the Central Nervous System (CNS), limbs and axial skeleton, IHH is involved in cartilage differentiation while $\mathrm{DHH}$ is strongly linked to the development of germline cells and Schwann cells [74]. The message from the Hedgehog receptors is downstream transmitted through the messenger molecules. Some proteins, such as Fused or Costal 2, have been reported to be part of the Hedgehog signalling, but most of the signalling proteins are still unknown and further research is required to find the downstream receptors pathway [70]. Hyperactivation of Hedgehog pathway has been linked to several types of cancer such as basal cell carcinomas, advanced prostate cancer [71], advanced gastric adenocarcinomas [74] and medulloblastomas [77].

Another pathway involved in growth, survival and stem cell self-renewal is the Wingless Drosophila melanogaster segment-polarity gene and Integrase-1 vertebrate homologue (Wnt) pathway. The members of the Wnt family consist of secreted lipoprotein ligands. By binding to various receptors, Wnt ligands trigger receptor intracellular signalling, switching off the GSK-3 $\beta$-dependent degradation pathway, which in turn enables $\beta$-catenin to accumulate in the cytosol and to translocate the nucleus to activate transcription of the Wnt target genes. Thus, in the active state, Wnts bind to receptors of the Frizzled and LRP5/6 co-receptors on the cell surface leading to $\beta$-catenin protein stabilization and triggering dislocation of the GSK-3 $\beta$ kinase from the APC/Axin/GSK-3 $\beta$-complex. In the active Wnt signals, $\beta$-catenin interplays with CK1 and the APC/Axin/GSK-3 $\beta$-complex, activating $\beta$-TrCP/SKP pathway that induces protein ubiquitination and proteasomal degradation.

The $\mathrm{Wnt} / \beta$-catenin is a well conserved pathway that regulates stem cell pluripotency during development. Aberrant Wnt signalling underlies a wide range of pathologies in humans, including cancer. Regarding its influence and implication in certain malignancies, the WNT pathway was incriminated to be linked to colorectal cancer, medulloblastoma and lymphoblastic leukemia. In most cases, the common denominator of the abnormal activity of this pathway is gene transcription activation by the protein $\beta$-catenin $[78,79]$. Several studies on genes activated in CSCs show that a large network of transcription factors (TFs) can be activated in these cells that are unlikely to ever occur in normal physiological conditions [80]. Among different CSC TFs, the Achaete-Scute Homolog 1 (ASCL1) gene is considered to be an upstream regulator of the Wnt signalling pathway. In a paper by 
Rheinbay et al. [80], ASCL1 was shown to activate Wnt pathway by repressing the negative regulator Dickkopf-related Protein 1 (DKK1) gene in glioblastoma (GBM) CSCs. It has been recently demonstrated that ASCL1 is crucial for GBM CSCs in vivo tumorgenicity [81].

Identified as a member of the Polycomb group, the BMI 1 pathway has been directly linked to self-renewal and differentiation of human stem cells. Its most significant effects have been associated with hematopoiesis, skeleton development and neural growth. BMI 1 deficient mice present an overwhelming depletion of nervous stem cells which translated into progressive post-natal neurological retardation and other significant defects. BMI 1 depletion also results in a significant reduction in self-renewal capacity of the HSCs [82].

BMI 1 over-expression and amplification have also been found in several types of malignancies, most notably in hematological ones such as leukemias or mantle cell lymphomas [83] due to its direct implication in self-renewal of hematopoietic stem cells. Other studies link the BMI 1 pathway to: gliomas [82], nasopharyngeal carcinomas [84] and ovarian cancer [85]. Recently, a link between the Hedgehog pathway and the BMI-1 gene has been found in several types of cancer, such as breast, basal cell carcinomas, medulloblastomas and esophageal cancers [86,87]. It has been suggested that in breast cancer the BMI-1 gene was present in the downstream signalling of the Hedgehog pathway and that activation of the same pathway determines an upregulation of the BMI-1 expression [88].

Cleton-Jensen et al. [89] have demonstrated that miRNAs are also implicated in emergence of CSCs from ESFTs. They were able to present a scenario where deregulation of miRNA in CSCs could be the cause of expression of several TFs that help generate and sustain tumorgenicity in CSC populations. Although miRNA repression is a key feature of malignant cells, there is little evidence of shared miRNA profiles between different types of cancer cells, this group considered that because different transcript expressions can be regulated by different miRNAs, it is easy to believe that CSCs derived from different types of tumors could survive by exploiting different miRNAs to regulate expression of their essential TFs. An example could be the ESFT and breast cancer CSCs that have been demonstrated to share the same miRNA profile for $c-M y c \mathrm{TF}$ regulation [90].

\section{Targeted Therapy against Cancer Stem Cells (CSCs)}

Although advances have been achieved in cancer therapy by using modern techniques and therapeutic approaches such as personalized targeted therapy, the solid tumor cells are generally resistant to treatment and patients continue to exhibit a poor prognosis [91]. The concept that tumor development and progression depends on the evolution of CSCs dramatically changes the possibilities in which cancer can be cured. This CSC population is very important in the tumor's malignant potential and response to therapy [2]. It is known that cancer therapy targeting the tumor cell bulk can produce a partial regression of the tumor, followed by the appearance of new tumor clones, developed from existing CSC population. Therefore, identification and targeting of neoplastic stem cells represents a major challenge in modern cancer therapy and researchers are trying to find new molecular therapies directed specifically against these cells (Figure 2). Thus, molecular therapies against CSCs were reported to be more effective, as they induce tumor regression by reducing the occurrence of new cancer cells (Figure 2) $[49,65]$. 
Many studies in clinically relevant cancer therapy are based on the hypothesis that there is a strong relationship between the tumor resistance to conventional therapy and the CSCs intrinsic mechanisms of resistance to conventional chemotherapeutic drugs and radiation therapy. Targeting CSCs or specific pathways responsible for radiation or drug resistance were reported to improve the treatment effect. CSCs harbor numerous intrinsic mechanisms of resistance to conventional chemotherapeutic drugs, radiation therapy, and novel tumor-targeting drugs that permit CSC survival of current cancer therapies and CSC-mediated initiation of tumor recurrence and metastasis [92-96].

In the last years, some insight regarding the intracellular and extracellular mechanisms that regulate stem cell division and differentiation has been provided. Different intrinsic and extrinsic factors were proposed to influence CSC expansion. These factors, present in CSCs, have been divided into the following categories: survival, differentiation, multidrug resistance, signal-transduction and oxidative stress factors [97]. New generation of cancer therapeutic drugs are designed to eliminate CSCs by interfering with the pathways mentioned above. These drugs have been examined in vivo and in vitro.

Figure 2. Targeted therapy against cancer stem cells. Therapeutics aimed at the general population of cancer cells has a limited impact on cancer stem cells (CSCs). In time, their regenerative and proliferative capabilities are responsible for the relapse. On the contrary, therapy aimed at the CSC population determines a destruction of the population responsible for differentiation and proliferation which in turn produces a much more pronounced and stable tumoral regression. The CSCs are displayed as the green, larger cells while the non-stem cells are depicted as the smaller, red with the skull and bones in the middle.

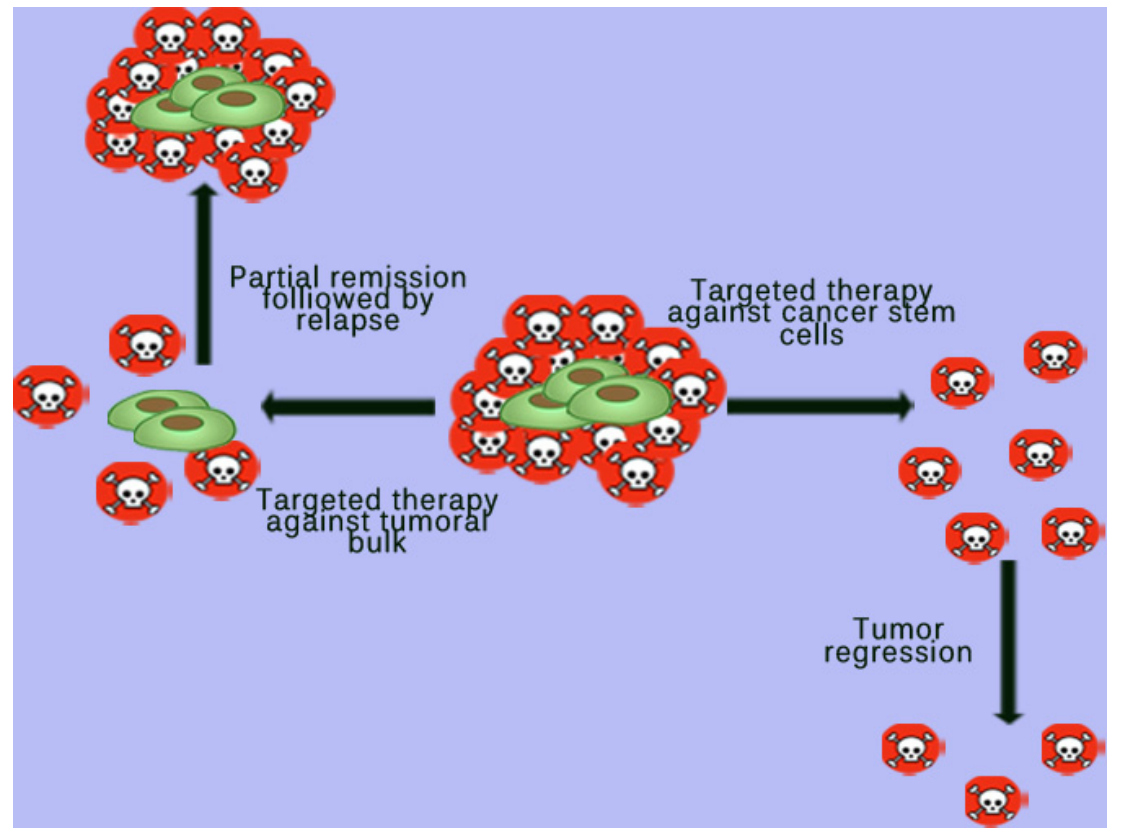

It has been speculated that some stem-cell signal pathways such as Wnt, Fibroblast Growth Factor (FGF), Notch, Hedgehog, and TGF $\beta /$ BMP (Bone Morphogenetic Protein) signaling play an important role in the pluripotent stem cells and CSCs homeostasis [98]. Using high throughput screening methods, several pharmaceutical companies developed drugs that specifically target these pathways. Inhibition of $\mathrm{Wnt} / \beta$-catenin signalling pathway by siRNA or by small molecule XAV939 was reported to induce cell death in several types of cancer cells $[99,100]$. Sonic Hedgehog signalling has also been 
evaluated as targeted pathway in regulating CSC growth in many cancer types. Some small-molecule modulators of hedgehog signaling have shown success in treating medulloblastoma, basal cell carcinoma, pancreatic cancer and prostate cancer [101]. In addition, blocking the Hedgehog signalling by siRNA chemosensitized the hepatocellular carcinoma cells to 5-fluorouracil (5-FU) treatment [102].

It is known that CSCs can be identified by several cell surface antigens such as CD133, CD90, CD44, OV6, and CD326 (EpCAM). EpCAM is considered an epithelial differentiation marker, however only when associated with stemness markers such as CD133, it can be used as a progenitor CSC marker in experiments.

Studies show that hepatic CSCs can be identified using several markers including CD133, CD24, CD44, CD90 and EpCAM [103]. A study by Chen et al. [104] demonstrated that CD133+, EpCAM+ cells, showed similar properties as tumor initiating cells (TICs) in Huh-7 cells, such as high differentiation capacity, increased potential of colony-formation, preferential expression of stem cell-related genes, MDR to some chemotherapeutics, more spheroid formation in cell cultures and higher tumorigenicity in NOD/SCID mice. Another study on pancreatic cancer stem-like cells demonstrated that isolated CD44+, CD133+, EpCAM+ cells of human pancreatic cancer behave as cancer stem-like cells, which show more aggressive behavior such as increased cell growth and migration, clonogenicity, and self-renewal capacity [105].

Thus, antibody-based therapeutic approaches targeting CD133, CD90, CD44, OV6, and CD326 are currently being developed [106,107].

Although a specific antibody may be effective in eradicating CSC, it is important to note that in most situations, this therapeutic method is not enough to eradicate the whole tumor. It has been hypothesized that tumor cells can escape the cytotoxic effect of specific antibodies by decreasing the expression of surface antigen, by developing resistance to cancer chemotherapeutic agents or by acquiring multiple mutations. For this reason, therapeutic approaches targeting CSCs in combination with traditional cancer therapies have been also used in preclinical experiments and in clinical trials [107].

Retinoic acid, a stem cell differentiation agent, has been used in combination chemotherapy in treatment of acute promyelocytic leukemia [108]. Inactivation of intracellular stem cell signalling pathways in combination with self-renewal of CSCs has also been experimented in vivo and in vitro [109,110].

Hermann and colleagues reported that a combined regime of a new hedgehog pathway inhibitor SIBI-C1 with Rapamycin and Gemcitabine yielded very promising results. The triple treatment completely depleted the CSCs population in primary human pancreatic cancer tissue xenografts having a profound effect on the tumoral stroma as well. In order to assess the effect on these therapeutic agents on normal stem cells, blood levels were followed throughout the study and compared to a standard gemcitabine regime. The triple combination treatment did not yield any significantly elevated side effects as it was initially expected to do [111].

In another study, Guofang Chen et al. demonstrated that administration of high levels of metformin in thyroid carcinomas inhibits growth of both thyroid carcinoma cells and CSCs. This effect was suggested to be related to Metformin's inhibitory effect on the insulin/IGF to 5' Adenosine monophosphate (AMP)-activated protein kinase (AMPK) and mTOR signalling pathways, which was present in both types of cells. Due to the over-expressed nature of the insulin/IGF and AMPK/mTOR receptors in both stand and CSCs as opposed to normal cells, the inhibitory effects were substantially different in the two populations [112]. 
A study performed by Chia-Hsin Chan et al. has shown that inhibition of the Skp2 protein, a ligase responsible for Akt-mediated aerobic glycolysis, resulted in a reduced self-renewal capacity of CSCs in prostate cancer. Inhibition of Skp2 also increased tumor cell sensitivity to chemotherapeutic agents. The therapeutic agent had shown a significantly higher affinity and consequential destructive effect for cancer cells in comparison to normal cells, where it showed a noticeably smaller effect over the viability of normal cells [113].

Even though chemotherapy is the treatment of choice against most types of cancers, its effectiveness is limited due to multi-drug resistance (MDR) developed by CSCs. There are three major mechanisms proposed in order to explain MDR, but the most investigated it is the efflux of cellular cytotoxic drugs, related to the over-expression of multifunctional efflux transporters, the ATP-binding cassette (ABC) proteins. ABC efflux pumps confer protection to CSCs, shielding them from the adverse effects of chemotherapeutic insult [90,114-116].

Two models have been proposed to explain MDR in CSCs; one suggests that after chemotherapy only $\mathrm{ABC}$ expressing CSCs are able to repopulate the tumor with newly formed CSCs or differentiated progenitor cells. The second model proposes that only CSCs that acquire drug resistance survive under the pressure of mutations and give rise to new and more aggressive drug-resistant cell phenotypes [114]. Therefore, chemoresistant CSCs are considered to be the Achilles' heel of cancer [117]. Some authors have identified two compounds: DECA-14 and rapamycin that selectively target neuroblastoma (NB) CSC, while having little effect on normal stem cells, preventing NB CSC self-renewal both in vitro and in vivo [5]. However, ongoing research is focusing on the efficacy of targeting multiple key $\mathrm{TF}$ that are responsible for CSCs' stemness [118].

Unfortunately, most of these new therapeutic approaches are not highly specific for CSCs, because the difference between the types of cell populations existing in the tumor is still difficult to find, since several signaling pathways are involved in regulation of cell proliferation of both CSCs and normal adult stem cells. This means that the percentage of abnormalities including certain distinctive features such as over expression of specific genes and/or receptors is still relatively small. Also, due to the nouvelle nature of therapies targeting CSC signalling pathways, many of the studies are still in preclinical phases of trials where the implications of above mentioned treatments on normal stem cells are difficult to assess. In addition, cancers can be triggered by multiple oncogenic mutations or CSC can suffer multiple mutations. For this reason, CSCs targeted therapy may be limited or may even fail and further research studies are needed to find better methods to specifically eliminate the CSC population.

\section{Conclusions}

Although most questions regarding the origin and certain features of CSCs remain unanswered, their existence within tumors is widely accepted. Also, more clinical and experimental data show that curative cancer therapy is effective only when CSC is completely eradicated. Current therapeutic approaches against cancer have been reported to control cell proliferation and tumor growth but they are not able to completely eradicate the tumor cell mass. Remaining cells often metastasize or recur in the same location. Chemo- and radio-therapy on the tumor bulk may lead to a better therapeutic response, but cannot provide a long-term complete remission of tumors. The role of CSCs in diagnosis 
and therapy of cancer has recently been the subject of intense research. Therefore, improving anti-cancer treatment response requires more accurate identification of the CSCs.

\section{Acknowledgments}

The authors acknowledge support by UEFISCDI Romania (Grant 134/2011).

\section{Conflicts of Interest}

The authors declare no conflict of interest.

\section{References}

1. Dick, J.E. Stem cell concepts renew cancer research. Blood 2008, 112, 4793.

2. Bonnet, D.; Dick, J.E. Human acute myeloid leukemia is organized as a hierarchy that originates from a primitive hematopoietic cell. Nat. Med. 1997, 3, 730-737.

3. Hope, K.J.; Jin, L.; Dick, J.E. Acute myeloid leukemia originates from a hierarchy of leukemic stem cell classes that differ in self-renewal capacity. Nat. Immunol. 2004, 5, 738-743.

4. Passegué, E.; Jamieson, C.H.M.; Ailles, L.E.; Weissman, I.L. Normal and leukemic hematopoiesis: Are leukemias a stem cell disorder or a reacquisition of stem cell characteristics? Proc. Natl. Acad. Sci. USA 2003, 100, 11842-11849.

5. Castelo-Branco, P.; Zhang, C.; Lipman, T.; Fujitani, M.; Hansford, L.; Clarke, I.; Harley, C.B.; Tressler, R.; Malkin, D.; Walker, E.; et al. Neural tumor-initiating cells have distinct telomere maintenance and can be safely targeted for telomerase inhibition. Clin. Cancer Res. 2011, 17, $111-121$.

6. Li, C.; Hynes, M.J.; Jing, J. Pancreatic cancer stem cells: New direction for pancreatic cancer treatment. Trends Bio/Pharm. Ind. 2010, 6, 34-40.

7. Zhang, S.; Balch, C.; Chan, M.W.; Lai, H.C.; Matei, D.; Schilder, J.M.; Yan, P.S.; Huang, T.H.; Nephew, K.P. Identification and characterization of ovarian cancer-initiating cells from primary human tumors. Cancer Res. 2008, 68, 4311-4320.

8. Miki, J.; Furusato, B.; Li, H.; Gu, Y.; Takahashi, H.; Egawa, S.; Sesterhenn, I.A.; McLeod, D.G.; Srivastava, S.; Rhim, J.S. Identification of putative stem cell markers, CD133 and CXCR4, in hTERT-immortalized primary nonmalignant and malignant tumor-derived human prostate epithelial cell lines and in prostate cancer specimens. Cancer Res. 2007, 67, 3153-3161.

9. Beier, D.; Hau, P.; Proescholdt, M.; Lohmeier, A.; Wischhusen, J.; Oefner, P.J.; Aigner, L.; Brawanski, A.; Bogdahn, U.; Beier, C.P. CD133(+) and CD133(-) glioblastoma-derived cancer stem cells show differential growth characteristics and molecular profiles. Cancer Res. 2007, 67, 4010-4015.

10. Bao, S.; Wu, Q.; McLendon, R.E.; Hao, Y.; Shi, Q.; Hjelmeland, A.B.; Dewhirst, M.W.; Bigner, D.D.; Rich, J.N. Glioma stem cells promote radioresistance by preferential activation of the DNA damage response. Nature 2006, 444, 756-760. 
11. Liu, G.; Yuan, X.; Zeng, Z.; Tunici, P.; Ng, H.; Abdulkadir, I.R.; Lu, L.; Irvin, D.; Black, K.L.; $\mathrm{Yu}$, J.S. Analysis of gene expression and chemoresistance of CD133+ cancer stem cells in glioblastoma. Mol. Cancer 2006, 5, 67.

12. Tirino, V.; Desiderio, V.; Paino, F.; de Rosa, A.; Papaccio, F.; Fazioli, F.; Pirozzi, G.; Papaccio, G. Human primary bone sarcomas contain CD133+ cancer stem cells displaying high tumorigenicity in vivo. FASEB J. 2011, 6, 2022-2030.

13. Suvà, M.L.; Riggi, N.; Stehle, J.C.; Baumer, K.; Tercier, S.; Joseph, J.M.; Suva, D.; Clement, V.; Provero, P.; Cironi, L.; et al. Identification of cancer stem cells in Ewing's sarcoma. Cancer Res. 2009, 69, 1776-1781.

14. Boveri, T. The Origin of Malignant Tumors; Williams and Wilkins: Baltimore, MD, USA, 1914.

15. Tyzzer, E.E. Tumor immunity. J. Cancer Res. 1916, 1, 125-156.

16. Muller, H.J. Radiation damage to the genetic material. Sci. Prog. 1951, 7, 33-59.

17. Berenblum, I.; Shubik, P. A new, quantitative, approach to the study of the stages of chemical carcinogenesis in the mouse's skin. Br. J. Cancer 1947, 1, 383-391.

18. Moolgavkar, S.H.; Knudson, A.G. Mutation and cancer: A model for human carcinogenesis. J. Natl. Cancer Inst. 1981, 66, 1037-1052.

19. Knudson, A.G., Jr. Hereditary cancers of man. Cancer Investig. 1983, 1, 187-193.

20. Knudson, A.G., Jr. Hereditary cancer, oncogene, and antioncogenes. Cancer Res. 1985, 45, 1437-1443.

21. Harbour, J.W.; Lai, S.L.; Whang-Peng, J.; Gazdar, A.F.; Minna, J.D.; Kaye, F.J. Abnormalities in structure and expression of the human retinoblastoma gene in SCLC. Science 1988, 24, $353-357$.

22. Lee, W.H.; Shew, J.Y.; Hong, F.D.; Sery, T.W.; Donoso, L.A.; Young, L.J.; Bookstein, R.; Lee, E.Y. The retinoblastoma susceptibility gene encodes a nuclear phosphoprotein associated with DNA binding activity. Nature 1987, 329, 642-645.

23. Nowell, P.C. The clonal evolution of tumor cell populations. Science 1976, 194, 23-28.

24. Hanahan, D.; Weinberg, R. The hallmarks of cancer. Cell 2000, 100, 57-70.

25. Weinberg, R.A. The Biology of Cancer; Taylor \& Francis: New York, NY, USA, 2006.

26. Triolo, V.A. Nineteenth century foundations of cancer research advances in tumor pathology, nomenclature, and theories of oncogenesis. Cancer Res. 1965, 25, 76-98.

27. Soto, A.M.; Sonnenschein, C. Emergentism as a default: Cancer as a problem of tissue organization. J. Biosci. 2005, 30, 103-118.

28. Feinberg, A.P.; Ohlsson, R.; Henikoff, S. The epigenetic progenitor origin of human cancer. Nat. Rev. Genet. 2006, 7, 21-33.

29. Rock, J.R.; Randell, S.H.; Hogan, B.L. Airway basal stem cells: A perspective on their roles in epithelial homeostasis and remodeling. Dis. Model. Mech. 2010, 3, 545-556.

30. Rock, J.R.; Onaitis, M.W.; Rawlins, E.L.; Lu, Y.; Clark, C.P.; Xue, Y.; Randell, S.H.; Hogan, B.L.M. Basal cells as stem cells of the mouse trachea and human airway epithelium. Proc. Natl. Acad. Sci. USA 2009, 106, 12771-12775.

31. Lapidot, T.; Sirard, C.; Vormoor, J.; Murdoch, B.; Hoang, T.; Caceres-Cortes, J.; Minden, M.; Paterson, B.; Caligiuri, M.A.; Dick, J.E. A cell initiating human acute myeloid leukaemia after transplantation into SCID mice. Nature 1994, 367, 645-648. 
32. Van Keymeulen, A.; Blanpain, C. Tracing epithelial stem cells during development, homeostasis, and repair. J. Cell Biol. 2012, 197, 575-584.

33. Moore, M.A.; Williams, N.; Metcalf, D. In vitro colony formation by normal and leukemic human hematopoietic cells: Characterization of the colony forming cells. J. Nat. Cancer. Inst. 1973, 50, 603-623.

34. Dalerba, P.; Cho, R.W.; Clarke, M.F. Cancer stem cells: Models and concepts. Ann. Rev. Med. 2007, 58, 267-284.

35. Shackleton, M.; Quintana, E.; Fearon, E.R.; Morrison, S.J. Heterogeneity in cancer: Cancer stem cells vs. clonal evolution. Cell 2009, 138, 822-829.

36. Dick, J.E. Breast cancer stem cells revealed. Proc. Natl. Acad. Sci. USA 2003, 100, 3547-3549.

37. Wicha, M.S.; Liu, S.; Dontu, G. Cancer stem cells: An old idea-A paradigm shift. Cancer Res. 2006, 66, 1883.

38. Sell, S. On the stem cell origin of cancer. Am. J. Pathol. 2010, 176, 2584-2594.

39. Pierce, G.B.; Dixon, F.J. The demonstration of teratogenesis by metamorphosis of multipotential cells. Cancer 1959, 12, 573-583.

40. Ushijima, T. Epigenetic field for cancerization. J. Biochem. Mol. Biol. 2007, 40, 142-150.

41. Ushijima, T. Detection and interpretation of altered methylation patterns in cancer cells. Nat. Rev. Cancer 2005, 5, 223-231.

42. Sell, S. Stem cells in hepato carcinogenesis. Cell Sci. Rev. 2006, 3, 1742-8130.

43. Rous, P. A sarcoma of the fowl transmissible by an agent separable from the tumor cells. J. Exp. Med. 1911, 13, 397-411.

44. Reya, T.; Morrison, S.J.; Clarke, M.F.; Weissman, I.L. Stem cells, cancer, and cancer stem cells Nature 2001, 414, 105-111.

45. Odoux, C.; Fohrer, H.; Hoppo, T.; Guzik, L.; Stolz, D.B.; Lewis, D.W.; Gollin, S.M.; Gamblin, T.C.; Geller, D.A.; Lagasse, E. A stochastic model for cancer stem cell origin in metastatic colon cancer. Cancer Res. 2008, 68, 6932-6941.

46. Fearon, E.R.; Vogelstein, B. A genetic model for colorectal tumorigenesis. Cell 1990, 61, 759-767.

47. Bapat, S.A. Evolution of cancer stem cells. Semin. Cancer Biol. 2007, 17, 204-213.

48. Chaffer, C.L.; Brueckmann, I.; Scheela, C. Normal and neoplastic nonstem cells can spontaneously convert to a stem-like state. Proc. Natl. Acad. Sci. USA 2011, 108, 7950-7955.

49. Dick, J.E. Looking ahead in cancer stem cell research. Nat. Biotechnol. 2009, 27, 44-46.

50. Quintana, E.; Shackleton, M.; Sabel, M.S.; Fullen, D.R.; Johnson, T.M.; Morrison, S.J. Efficient tumor formation by single human melanoma cells. Nature 2008, 456, 593-598.

51. Dingli, D.; Traulsen, A.; Pacheco, J.M. Stochastic dynamics of hematopoietic tumor stem cells. Cell Cycle 2007, 6, 461-466.

52. Vermeulen, L.; de Sousa E Melo, F.; van der Heijden, M. Cameron, K.; de Jong, J.H.; Borovski, T.; Tuynman, J.B.; Todaro, M.; Merz, C.; Rodermond, H.; et al. Wnt activity defines colon cancer stem cells and is regulated by the microenvironment. Nat. Cell Biol. 2010, 12, 468-476.

53. Roesch, A.; Fukunaga-Kalabis, M.; Schmidt, E.C.; Zabierowski, S.E.; Brafford, P.A.; Vultur, A.; Basu, D.; Gimotty, P.; Vogt, T.; Herlyn, M. A temporarily distinct subpopulation of slow-cycling melanoma cells is required for continuous tumor growth. Cell 2010, 141, 583-594. 
54. Giannoni, E.; Bianchini, F.; Masieri, L.; Serni, S.; Torre, E.; Galorini, L.; Chiarugi, P. Reciprocal activation of prostate cancer cells and cancer-associated fibroblasts stimulates epithelial-mesenchymal transition and cancer stemness. Cancer Res. 2010, 70, 6945-6956.

55. Fiaschi, T.; Marini, A.; Giannoni, E.; Taddei, M.L.; Gandellini, P.; de Donatis, A.; Lanciotti, M.; Serni, S.; Cirri, P.; Chiarugi, P. Reciprocal metabolic reprogramming through lactate shuttle coordinately influences tumor-stroma interplay. Cancer Res. 2012, 72, 5130-5140.

56. Fiaschi, T.; Giannoni, E.; Taddei, M.L.; Cirri, P.; Marini, A.; Pintus, G.; Nativi, C.; Richichi, B.; Scozzafava, A.; Carta, F.; et al. Carbonic anhydrase IX from cancer associated fibroblasts drives epithelial-mesenchymal transition in prostate carcinoma cells. Cell Cycle 2013, 12, 1791-1801.

57. Hay, E.D. An overview of epithelio-mesenchymal transformation. Acta Anat. 1995, 154, 8-20.

58. Thiery, J.P.; Sleeman, J.P. Complex networks orchestrate epithelial-mesenchymal transitions. Nat. Rev. Mol. Cell Biol. 2006, 7, 131-142.

59. Mani, S.A.; Guo, W.; Liao, M.J. The epithelial-mesenchymal transition generates cells with properties of stem cells. Cell 2008, 133, 704-715.

60. Giannoni, E.; Bianchini, F.; Calorini, L.; Chiarugi, P. Cancer associated fibroblasts exploit reactive oxygen species through a proinflammatory signature leading to epithelial mesenchymal transition and stemness. Antioxid. Redox. Signal. 2011, 14, 2361-2371.

61. Cirri, P.; Chiarugi, P. Cancer-associated-fibroblasts and tumor cells: A diabolic liaison driving cancer progression. Cancer Metastasis Rev.2012, 31, 195-208.

62. Piccirillo, S.G.; Reynolds, B.A.; Zanetti, N.; Lamorte, G.; Binda, E.; Broggi, G. Bone morphogenetic proteins inhibit the tumorigenic potential of human brain tumor-initiating cells. Nature 2006, 444, 761-765.

63. Al-Hajj, M.; Wicha, M.S.; Benito-Hernandez, A. Prospective identification of tumorigenic breast cancer cells. Proc. Natl. Acad. Sci. USA 2003, 100, 3983-3988.

64. Singh, S.K.; Hawkins, C.; Clarke, I.D. Identification of human brain tumor initiating cells. Nature 2004, 432, 396-401.

65. Cho, R.W.; Clarke, M.F. Recent advances in cancer stem cells. Curr. Opin. Genet. Dev. 2008, $18,48-53$.

66. Bloushtain-Qimron, N.; Yao, J.; Snyder, E.L.; Shipitsin, M.; Campbell, L.L.; Mani, S.A. Cell type-specific DNA methylation patterns in the human breast. Proc. Natl. Acad. Sci. USA 2008, 105, 14076-14081.

67. Shipitsin, M.; Campbell, L.L.; Argani, P.; Weremowicz, S.; Bloushtain-Qimron, N.; Yao, J.; Nikolskaya, T.; Serebryiskaya, T.; Beroukhim, R.; Hu, M.; et al. Molecular definition of breast tumor heterogeneity. Cancer Cell 2007, 11, 259-273.

68. Kondo, T.; Setoguchi, T.; Taga, T. Persistence of a small subpopulation of cancer stem-like cells in the C6 glioma cell line. Proc. Natl. Acad. Sci. USA 2004, 101, 781-786.

69. Zheng, X.; Shen, G.; Yang, X.; Liu, W. Most C6 cells are cancer stem cells: Evidence from clonal and population analyses. Cancer Res. 2007, 67, 3691-3697.

70. Fukaya, R.; Ohta, S.; Yamaguchi, M.; Fujii, H.; Kawakami, Y. Isolation of cancer stem-like cells from a side population of a human glioblastoma cell line, SK-MG-1. Cancer Lett. 2010, $291,150-157$. 
71. Qiang, L.; Yang, Y.; Ma, Y.J.; Chen, F.H.; Zhang, L.B. Isolation and characterization of cancer stem like cells in human glioblasoma cell lines. Cancer Lett.2009, 279, 13-21.

72. Bale, A.E.; Yu, K.P. The hedgehog pathway and basal cell carcinomas. Hum. Mol. Genet. 2001, 10, 757-762.

73. Sheng, T.; Li, C.; Zhang, X.; Chi, S.; He, N.; Chen, K.; McCormick, F.; Gatalica, Z.; Xie, J. Activation of the hedgehog pathway in advanced prostate cancer. Mol. Cancer 2004, 3, 29.

74. Takebe, N.; Harris, P.J.; Warren, R.Q.; Ivy, S.P. Targeting cancer stem cells by inhibiting Wnt, notch, and Hedgehog pathways. Nat. Rev. Clin. Oncol. 2011, 8, 97-106.

75. Rizzo, P.; Osipo, C.; Foreman, K.; Golde, T.; Osborne, B.; Miele, L. Rational targeting of Notch signalling in cancer. Oncogene 2008, 27, 5124-5131.

76. Ma, X.; Chen, K.; Huang, S.; Zhang, X.; Adegboyega, P.A.; Evers, B.M. Frequent activation of the hedgehog pathway in advanced gastric adenocarcinomas. Carcinogenesis 2005, 26, 1698-1705.

77. Taylor, M.D.; Liu, L.; Raffel, C.; Hui, C.C.; Mainprize, T.G.; Zhang, X. Mutations in SUFU predispose to medulloblastoma. Nat. Genet. 2002, 31, 306-310.

78. Polakis, P. Wnt signaling and cancer. Genes Dev. 2000, 14, 1837-1851.

79. Pardal, R.; Clarke, M.F.; Morrison, S.J. Applying the principles of stem-cell biology to cancer. Nat. Rev. Cancer 2003, 3, 895-902.

80. Rheinbay, E.; Suvà, M.L. An aberrant transcription factor network essential for Wnt signaling and stem cell maintenance in glioblastoma. Cell Rep. 2013, 3, 1567-1579.

81. Zhang, N.; Wei, P.; Gong, A. FoxM1 Promotes $\beta$-catenin nuclear localization and controls Wnt target-gene expression and glioma tumorigenesis. Cancer Cell 2011, 20, 427-442.

82. Godlewski, J.; Nowicki, M.O.; Bronisz, A.; Williams, S.; Otsuki, A.; Nuovo, G. Targeting of the Bmi-1 oncogene/stem cell renewal factor by microRNA-128 inhibits glioma proliferation and self-renewal. Cancer Res. 2008, 68, 9125-9130.

83. Beà, S.; Tort, F.; Pinyol, M.; Puig, X.; Hernández, L.; Hernández, S. BMI-1 gene amplification and over-expression in hematological malignancies occur mainly in mantle cell lymphomas. Cancer Res. 2001, 61, 2409-2412.

84. Song, L.B.; Zeng, M.S.; Liao, W.T.; Zhang, L.; Mo, H.Y.; Liu, W.L. Bmi-1 is a novel molecular marker of nasopharyngeal carcinoma progression and immortalizes primary human nasopharyngeal epithelial cells. Cancer Res. 2006, 66, 6225-6232.

85. Bhattacharya, R.; Nicoloso, M.; Arvizo, R.; Wang, E.; Cortez, A.; Rossi, S. MiR-15a and MiR-16 control Bmi-1 expression in ovarian cancer. Cancer Res. 2009, 69, 9090-9095.

86. Lowell, E.M.; Westerman, B.A.; Ermilov, A.N. BMII is required for Hedgehog pathwayDriven medulloblastoma expansion. Neoplasia 2008, 10, 1343-1349.

87. Fujiwara, Y.; Yoshikawa, R.; Tao, L.; Tsujimura, T.; Sasako, M. Stemness signature of BMI1 and clinical outcome in esophageal cancer patients undergoing neoadjuvant chemoradiotherapy. J. Clin. Oncol. 2009, 27, 4572.

88. Jiang, L.; Li, J.; Song, L. Bmi-1, stem cells and cancer. Acta Biochim. Biophys. Sin. 2009, 41, 527-534.

89. Cleton-Jansen, A.M.; Anninga, J.K.; Briaire-de Bruijn, I.H.; Romeo, S. Profiling of high-grade central osteosarcoma and its putative progenitor cells identifies tumorigenic pathways. Br. J. Cancer 2009, 101, 1909-1918. 
90. Yu, F.; Yao, H.; Zhu, P.; Zhang, X. Let-7 regulates self-renewal and tumorigenicity of breast cancer cells. Cell 2007, 131, 1109-1123.

91. Carapancea, M.; Alexandru, O.; Fetea, A.S.; Dragutescu, L.; Castro, J.; Georgescu, A.; Popa-Wagner, A. Growth factor receptors signalling in glioblastoma cells: Therapeutic implications. J. Neurooncol. 2009, 92, 137-147.

92. Moitra, K.; Lou, H.; Dean, M. Multidrug efflux pumps and cancer stem cells: Insights into multidrug resistance and therapeutic development. Clin. Pharmacol. Ther. 2011, 89, 491-502.

93. Vermeulen, L.; de Sousa E Melo, F.; Richel, D.J.; Medema, J.P. The developing cancer stem-cell model: Clinical challenges and opportunities. Lancet Oncol. 2012, 13, e83-e89.

94. Zhou, B.B.; Zhang, H.; Damelin, M.; Geles, K.G.; Grindley, J.C.; Dirks, P.B. Tumor-initiating cells: Challenges and opportunities for anticancer drug discovery. Nat. Rev. Drug Discov. 2009, 8, 806-823.

95. Naujokat, C.; Steinhart, R. Salinomycin as a drug for targeting human cancer stem cells. J. Biomed. Biotechnol. 2012, 2012, 950658.

96. Cordon-Cardo, C. Suppression of acquired docetaxel resistance in prostate cancer through depletion of Notch- and Hedgehog-dependent tumor-initiating cells. Cancer Cell 2012, 22, 373-388.

97. Rosen, J.M.; Jordan, C.T. The increasing complexity of the cancer stem cell paradigm. Science 2009, 324, 1670.

98. Katoh, M. Network of Wnt and other regulatory signalling cascades in pluripotent stem cells and cancer stem cells. Curr. Pharm. Biotechnol. 2011, 12, 160-170.

99. Zeng, G.; Apte, U.; Cieply, B. siRNA-mediated $\beta$-catenin knockdown in human hepatoma cells results in decreased growth and survival. Neoplasia 2007, 9, 951-959.

100. Adler, E.M. Inhibiting Wnt signalling. Sci. Signal. 2009, 2, ec326.

101. Stanton, B.Z.; Peng, L.F. Small-molecule modulators of the Sonic Hedgehog signalling pathway. Mol. Biosyst. 2010, 6, 44-54.

102. Wang, Q.; Huang, S.; Yang, L.; Zhao, L.; Yin, Y.; Liu, Z. Down-regulation of sonic hedgehog signaling pathway activity is involved in 5-fluorouracil-induced apoptosis and motility inhibition in Hep3B cells. Acta Biochim. Biophys. Sin. 2008, 40, 819-829.

103. Yamashita, T.; Ji, J.; Budhu, A.; Forgues, M.; Yang W. EpCAM-positive hepatocellular carcinoma cells are tumor - initiating cells with stem/progenitor cell features. Gastroenterology 2009, 136, 1012-1024.

104. Chen, Y.; Yu, D.; Zhang, H.; He, H.; Zhang, C. CD133(+)EpCAM(+) phenotype possesses more characteristics of tumor initiating cells in hepatocellular carcinoma Huh7 cells. Int. J. Biol. Sci. 2012, 8, 992-1004.

105. Bin, B.; Asfar, A.; Amro, A.; Aamir, A. Pancreatic cancer stem-like cells display aggressive behavior mediated via activation of FoxQ1. J. Biol. Chem. 2014, doi:10.1074/jbc.M113.532887.

106. Zhu, Z.; Hao, X.; Yan, M.; Yao, M.; Ge, C.; Gu, J.; Li, J. Cancer stem/progenitor cells are highly enriched in CD133+CD44+ population in hepatocellular carcinoma. Int. J. Cancer 2010, 126, 2067-2078.

107. Kurtz, J.E.; Dufour, P. Adecatumumab: An anti-EpCAM monoclonal antibody, from the bench to the bedside. Expert Opin. Biol. Ther. 2010, 10, 951-958. 
108. Motohashi, T.; Aoki, H.; Chiba, K. Multipotent cell fate of neural crest-like cells derived from embryonic stem cells. Stem Cells 2007, 25, 402-410.

109. O’Brien, C.A.; Kreso, A.; Jamieson, C.H. Cancer stem cells and self-renewal. Clin. Cancer Res. 2010, 16, 3113-3120.

110. Alison, M.R.; Lim, S.M.; Nicholson, L.J. Cancer stem cells: Problems for therapy? J. Pathol. 2010, 223, 148-162.

111. Hermann, P.C.; Trabulo, S.M.; Sainz, B., Jr.; Balic, A.; Garcia, E.; Hahn, S.A. Multimodal treatment eliminates cancer stem cells and leads to long-term survival in primary human pancreatic cancer tissue xenografts. PLoS One 2013, 8, e66371.

112. Chen, G.; Xu, S.; Renko, K.; Derwahl, M. Metformin inhibits growth of thyroid carcinoma cells, suppresses self-renewal of derived cancer stem cells, and potentiates the effect of chemotherapeutic agents. J. Clin. Endocrin. Metab. 2012, 97, 1-11.

113. Chan, C.H.; Morrow, J.K.; Li, C.F.; Gao, Y.; Jin, G.; Moten, A. Pharmacological inactivation of Skp2 SCF ubiquitin ligase restricts cancer stem cell traits and cancer progression. Cell 2013, 154, 556-568.

114. Alisi, A.; Cho, W.C.; Locatelli, F.; Fruci, D. Multidrug resistance and cancer stem cells in neuroblastoma and hepatoblastoma. Int. J. Mol. Sci. 2013, 14, 24706-24725.

115. Gottesman, M.M. Mechanisms of cancer drug resistance. Ann. Rev. Med. 2002, 53, 615-627.

116. Ullah, M.F. Cancer multidrug resistance (MDR): A major impediment to effective chemotherapy. Asian Pac. J. Cancer Prev. 2008, 9, 1-6.

117. Science Daily. Available online: http://www.sciencedaily.com/releases/2012/09/120910122114.htm (accessed on 10 October 2013).

118. Ruck, P.; Xiao, J.C.; Pietsch, T.; von Schweinitz, D.; Kaiserling, E. Hepatic stem-like cells in hepatoblastoma: Expression of cytokeratin 7, albumin and oval cell associated antigens detected by OV-1 and OV-6. Histopathology 1997, 31, 324-329.

(C) 2014 by the authors; licensee MDPI, Basel, Switzerland. This article is an open access article distributed under the terms and conditions of the Creative Commons Attribution license (http://creativecommons.org/licenses/by/3.0/). 